\title{
Quasi-One-Dimensional Polarized Fermi Superfluids
}

\author{
Meera M. Parish, ${ }^{1,2, *}$ Stefan K. Baur, ${ }^{3}$ Erich J. Mueller, ${ }^{3}$ and David A. Huse ${ }^{1}$ \\ ${ }^{1}$ Department of Physics, Princeton University, Princeton, New Jersey 08544, USA \\ ${ }^{2}$ Princeton Center for Theoretical Physics, Princeton University, Princeton, New Jersey 08544, USA \\ ${ }^{3}$ Laboratory for Atomic and Solid State Physics, Cornell University, Ithaca New York 14853, USA
}

(Received 17 September 2007; published 18 December 2007)

\begin{abstract}
We calculate the zero-temperature $(T=0)$ phase diagram of a polarized two-component Fermi gas in an array of weakly coupled parallel one-dimensional (1D) "tubes" produced by a two-dimensional optical lattice. Increasing the lattice strength drives a crossover from three-dimensional (3D) to 1D behavior, stabilizing the Fulde-Ferrell-Larkin-Ovchinnikov (FFLO) modulated superfluid phase. We argue that the most promising regime for observing the FFLO phase is in the quasi-1D regime, where the atomic motion is largely $1 \mathrm{D}$ but there is weak tunneling in the other directions that stabilizes long-range order. In the FFLO phase, we describe a phase transition where the quasiparticle spectrum changes from gapless near the $3 \mathrm{D}$ regime to gapped in quasi-1D.
\end{abstract}

DOI: $10.1103 /$ PhysRevLett.99.250403

Recent experiments on ultracold ${ }^{6} \mathrm{Li}$ have probed polarized, two-component Fermi gases as a function of interatomic interaction strength and spin population imbalance [1]. These experiments have focused on the unitarity regime, where the $s$-wave scattering length is large and the scattering properties are universal. At low temperatures, they have seen phase separation between an unpolarized or weakly polarized superfluid phase and a highly polarized normal phase. Future experiments hope to observe the elusive FFLO spatially-modulated superfluid phase, first predicted in magnetized superconductors over 40 years ago [2,3], and having realizations in other systems ranging from heavy-fermion superconductors [4] to quark matter [5]. While the FFLO phase is expected to exist in trapped $3 \mathrm{D}$ gases for small polarizations and weak attractive interactions [6], it only occupies a small part of the $T=0$ phase diagram [7], and this region is diminished with increasing temperature [8]. Here we theoretically explore how a twodimensional (2D) optical lattice can enlarge the region of stability of the FFLO phase, paving the way for its observation.

Although a 3D simple cubic optical lattice may also enhance the FFLO phase [9], we argue that a 2D optical lattice, which permits free motion in one direction, is more effective. Analogous to charge density wave instabilities, the instability of the normal state to FFLO is due to a Fermi surface "nesting", which is enhanced in 1D. By increasing the intensity of the optical lattice, one can tune the singleatom dispersion from $3 \mathrm{D}$ to $1 \mathrm{D}$, a scenario which is readily achieved experimentally [10].

As revealed by the Bethe ansatz [11,12], the exact $T=0$ phase diagram of the 1D polarized Fermi gas displays four phases: unpolarized superfluid (SF), FFLO, fully-polarized normal, and vacuum, characterized by the densities of the two species and by algebraic order. Unlike 3D, in 1D all of the phase transitions are continuous and the FFLO phase occurs at all nonzero partial polarizations for any strength of the attractive interaction [12]. Furthermore, at the phase
PACS numbers: 05.30.Fk, 03.75.Hh, 03.75.Ss, 74.20.- $\mathrm{z}$

boundary between polarized and unpolarized phases, the SF phase has the lower density in $1 \mathrm{D}$, which is opposite to the situation in 3D. In a trap, the spatial structure is therefore inverted: in $1 \mathrm{D}$ one has a central FFLO region surrounded by SF, for small polarizations. During the crossover from $1 \mathrm{D}$ to $3 \mathrm{D}$, we find a regime where the phase sequence moving from the center to the edge of the trap is the quasi-1D FFLO, then a shell of SF, then the more 3Dlike polarized normal.

We consider a gas of fermionic atoms in two different hyperfine states (labeled by $\sigma=\uparrow, \downarrow= \pm 1$ ) confined by a smooth trapping potential $V(\mathbf{r})$ and an optical lattice potential such as $V_{L}(\mathbf{r})=-U[\cos (2 \pi x / b)+\cos (2 \pi y / b)]$, with lattice spacing $b$ and depth $U$, which breaks the cloud into an array of tubes aligned along the $z$ direction. In the local density approximation (LDA), the properties at location $\mathbf{r}$ depend on the local chemical potentials $\mu_{\sigma}(\mathbf{r}) \equiv$ $\mu_{\sigma}-V(\mathbf{r})$ in the same way that a spatially uniform system does. Within LDA, which should be valid for a wide enough trap, the pattern of phases in a trap can be read off from the phase diagram of the uniform system by tracing the spatial variation of $\mu \equiv\left(\mu_{\uparrow}+\mu_{\downarrow}\right) / 2$, while holding the difference $h \equiv\left(\mu_{\uparrow}-\mu_{\uparrow}\right) / 2$ fixed.

To produce the uniform phase diagram we study the untrapped system in a uniform lattice with $N_{x} \times N_{y}$ tubes, each of length $L_{z}$ in the $z$ direction. For a sufficiently low density and strong enough lattice, the $x y$ motion is well approximated by a one-band tight-binding model with single-atom dispersion

$$
\epsilon_{\mathbf{k}}=\frac{k_{z}^{2}}{2 m}+2 t\left[2-\cos \left(k_{x} b\right)-\cos \left(k_{y} b\right)\right],
$$

where $t$ is the hopping (related to $U$ and $b$ as in [13]), $m$ is the atomic mass, and we use $\hbar=1$. The Brillouin zone of the $x y$ motion is $\left|k_{x}\right|,\left|k_{y}\right| \leq \pi / b$, while $k_{z}$ is unconstrained. For energies well above the $x y$ bandwidth $\epsilon_{\mathbf{k}} \gg 8 t$, the dispersion is 1D-like. For low energies $\epsilon_{\mathbf{k}} \ll t$, the dispersion is 3D-like, and can be made isotropic if we rescale the 
momenta $\left\{k_{x}, k_{y}, k_{z}\right\} \mapsto\left\{b \sqrt{2 t} k_{x}, b \sqrt{2 t} k_{y}, k_{z} / \sqrt{m}\right\}$. This single-band, tight-binding regime is accessed experimentally by working in a regime with $t, \varepsilon_{F \sigma} \ll \sqrt{U / m b^{2}}$, where $\varepsilon_{F \sigma}=\left(\pi n_{\sigma} b^{2}\right)^{2} / 2 m$ is the 1D Fermi energy for each species of density $n_{\sigma}$ (corresponding to the $1 \mathrm{D}$ density $n_{\sigma} b^{2}$ per tube in the optical lattice).

Since the ${ }^{6} \mathrm{Li}$ experiments use highly dilute gases with a wide Feshbach resonance, the interactions can be modeled by a contact interaction, giving a Hamiltonian,

$$
\begin{aligned}
\hat{H}-\mu_{\uparrow} \hat{N}_{\uparrow}-\mu_{\downarrow} \hat{N}_{\downarrow}= & \sum_{\mathbf{k}} \sum_{\sigma=\uparrow, \downarrow}\left(\epsilon_{\mathbf{k}}-\mu_{\sigma}\right) c_{\mathbf{k} \sigma}^{\dagger} c_{\mathbf{k} \sigma} \\
& +\frac{g}{L_{z} N_{x} N_{y}} \sum_{\mathbf{k}, \mathbf{k}^{\prime}, \mathbf{q}} c_{\mathbf{k} \uparrow}^{\dagger} c_{\mathbf{k}^{\prime} \downarrow}^{\dagger} c_{\mathbf{k}^{\prime}+\mathbf{q} \downarrow} c_{\mathbf{k}-\mathbf{q} \uparrow} .
\end{aligned}
$$

Solving the 3D scattering problem in a single harmonic tube of transverse size $a_{\perp}=\sqrt{1 / m \omega_{\perp}}$, one finds [14]:

$$
\frac{1}{g}=-\frac{m a_{1 D}}{2} \equiv \frac{m a_{\perp}}{2}\left(\frac{a_{\perp}}{a}-C\right),
$$

where $a$ is the 3D $s$-wave scattering length, arising from the short-range interatomic potential. Thus, we have an attractive 1D interaction when $a_{\perp} / a<C \simeq 1.4603 / \sqrt{2}$. Defining the 1D two-body binding energy $\varepsilon_{B}=g^{2} \mathrm{~m} / 4$, we can fully parameterize the $T=0$ phase diagram of the uniform system with three dimensionless quantities: $t / \varepsilon_{B}$, $\mu / \varepsilon_{B}$ and $h / \varepsilon_{B}[15]$.

We calculate the $T=0$ phase diagram within meanfield theory, which captures most of the qualitative features of the phase diagram as we move between the $1 \mathrm{D}$ and 3D regimes. However, we know by comparison to the exact solution that this mean-field approximation does miss some features of the 1D limit, as we note below.

We begin at $h=0$ where there are only two phases: SF and the vacuum (see Fig. 1). In the 1D limit (small $t$ ) there is a two-atom bound state with binding energy $E_{B}$, where clearly $E_{B}=\varepsilon_{B}$ when $t=0$. These bosonic pairs enter the

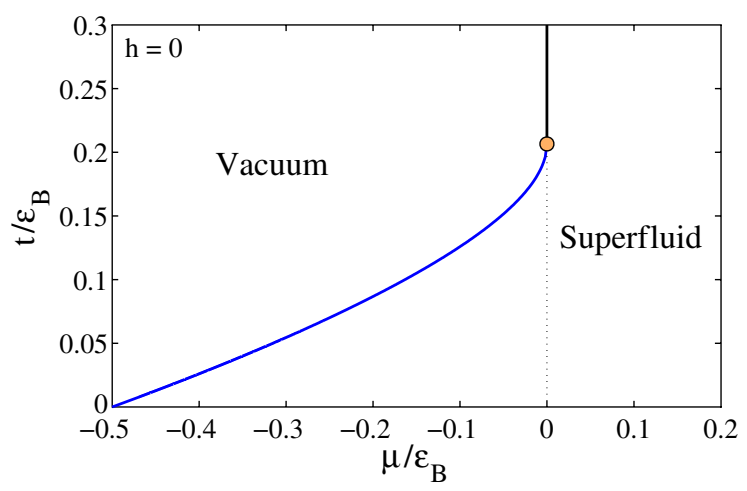

FIG. 1 (color online). Phase diagram for $h=0$. For $t / \varepsilon_{B}$ below the filled circle, there is a two-atom bound state, and the resulting bosonic pairs enter the system as a BEC as $\mu$ is increased through the solid line (given by $\mu=-E_{B} / 2$ ). For $t / \varepsilon_{B}$ above the filled circle we are always in the BCS regime. system and form a Bose-Einstein condensate (BEC) for $\mu>-E_{B} / 2$. Increasing the density further brings the system through a density-driven BEC-BCS crossover, similar to excitonic systems [16], where $\mu / E_{B} \gg 1$ defines the weak-coupling BCS limit. Making the system more 3D, by increasing $t$, reduces both $E_{B}$ and the BEC regime. For $t / \varepsilon_{B}>0.2066$ there is no two-atom bound state and thus only the BCS regime.

At finite $h$ we must also consider the FFLO superfluid phase, where the Cooper pairs condense with nonuniform pairing order parameter (gap)

$$
\begin{aligned}
\Delta(z) & =-\frac{g}{L_{z} N_{x} N_{y}} \sum_{\mathbf{k}, q_{z}} e^{i q_{z} z\left\langle c_{-\mathbf{k}+q_{z} \hat{\mathbf{z}} / 2 \downarrow} c_{\mathbf{k}+q_{z} \hat{\mathbf{z}} / 2 \uparrow}\right\rangle} \\
& =-\frac{g}{N_{x} N_{y}} \sum_{l, \mathbf{k}_{\perp}} u_{l, \mathbf{k}_{\perp}}(z) v_{l, \mathbf{k}_{\perp}}^{*}(z) f\left(E_{l, \mathbf{k}_{\perp}}\right),
\end{aligned}
$$

where $f(x)$ is the zero-temperature Fermi function, $\mathbf{k}_{\perp}$ is the momentum in the $x y$ plane, $l$ labels the quasiparticle modes, and the energies or coherence factors obey the Bogoliubov-de Gennes (BdG) equations [17]

$$
\left(\begin{array}{cc}
h_{0 \uparrow} & \Delta(z) \\
\Delta^{*}(z) & -h_{0 \downarrow}
\end{array}\right)\left(\begin{array}{c}
u_{l, \mathbf{k}_{\perp}}(z) \\
v_{l, \mathbf{k}_{\perp}}(z)
\end{array}\right)=E_{l, \mathbf{k}_{\perp}}\left(\begin{array}{c}
u_{l, \mathbf{k}_{\perp}}(z) \\
v_{l, \mathbf{k}_{\perp}}(z)
\end{array}\right),
$$

with $\quad h_{0 \sigma}=-\frac{\partial_{z}^{2}}{2 m}+2 t\left[2-\cos \left(k_{x} b\right)-\cos \left(k_{y} b\right)\right]+$ $g n_{-\sigma}(z)-\mu$. The densities in the Hartree term are $n_{\uparrow}(z)=\left(1 / N_{x} N_{y}\right) \sum_{l, \mathbf{k}_{\perp}}\left|u_{l, \mathbf{k}_{\perp}}(z)\right|^{2} f\left(E_{l, \mathbf{k}_{\perp}}\right) \quad$ and $\quad n_{\downarrow}(z)=$ $\left(1 / N_{x} N_{y}\right) \sum_{l, \mathbf{k}_{\perp}}\left|v_{l, \mathbf{k}_{\perp}}(z)\right|^{2} f\left(-E_{l, \mathbf{k}_{\perp}}\right)$. The grand potential is [18]

$$
\begin{aligned}
\Omega= & -N_{x} N_{y} \int d z\left[\frac{|\Delta(z)|^{2}}{g}+g n_{\uparrow}(z) n_{\downarrow}(z)\right] \\
& +\operatorname{tr}\left[\frac{h_{0 \uparrow}+h_{0 \downarrow}}{2}\right]+\sum_{l, \mathbf{k}_{\perp}, \sigma} \frac{E_{l, \mathbf{k}_{\perp}}+\sigma h}{2} f\left(E_{l, \mathbf{k}_{\perp}}+\sigma h\right),
\end{aligned}
$$

where the sum includes both positive and negative energy eigenvalues. The simplest ansatz for the FFLO phase is the Fulde-Ferrell one-plane wave state $\Delta(z)=\Delta_{\mathrm{FF}} e^{i q z}$ [2]. Here, $E_{l, \mathbf{k}_{\perp}}$ reduce to $E_{\mathbf{k} \pm}= \pm \sqrt{\left(\xi_{+}^{\dagger}+\xi_{-}^{\downarrow}\right)^{2} / 4+\Delta^{2}}+$ $\left(\xi_{+}^{\dagger}-\xi_{-}^{\downarrow}\right) / 2$, with $\xi_{ \pm}^{\sigma}=\epsilon_{\mathbf{k} \pm q \hat{\mathbf{z}} / 2}-\mu+g n_{-\sigma}$ and we can then minimize Eq. (6) directly. This state is a good approximation in the limit $\Delta_{F F} \rightarrow 0$. Indeed, one can show that the second-order transition to the normal phase occurs at single wave vector $q$ when [19]

$$
-\frac{1}{g}=\frac{1}{N_{x} N_{y} L_{z}} \sum_{\mathbf{k}} \frac{1-f\left(\xi_{+}^{\dagger}-h\right)-f\left(\xi_{-}^{\downarrow}+h\right)}{\xi_{+}^{\uparrow}+\xi_{-}^{\downarrow}} .
$$

The locus of this transition is illustrated in Fig. 2.

Larkin and Ovchinnikov [3] showed that the energy is lower if the Cooper pairs condense in a standing wave, with $\Delta(z)=\Delta_{\mathrm{LO}} \cos (q z)$ when the gap is small. More generally, $\Delta(z)$ is a real periodic function of $z$. When the coherence length $\xi$ is small compared to $1 / q$, this state consists 


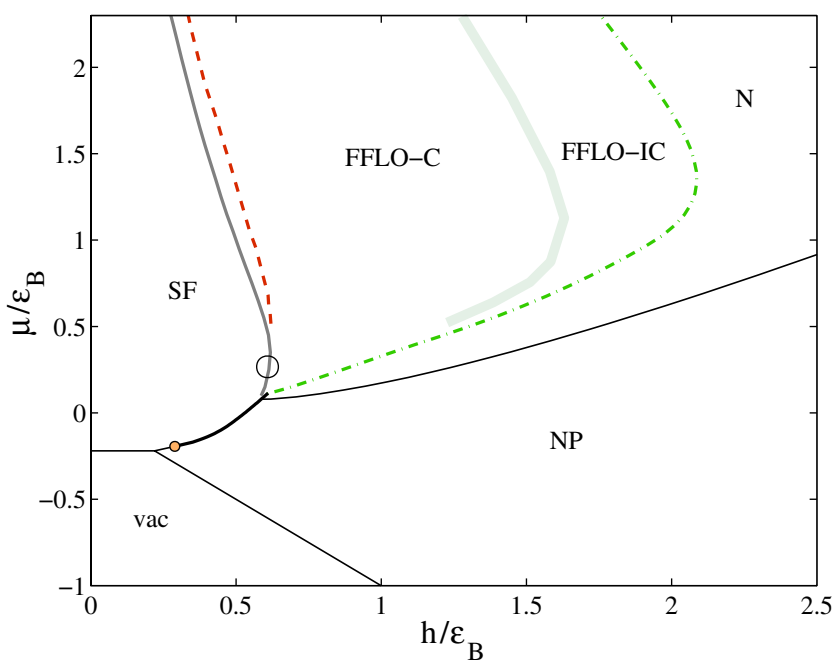

FIG. 2 (color online). Slice of the mean-field phase diagram taken at $t / \varepsilon_{B}=0.08$. The phases shown include the unpolarized superfluid (SF), partially-polarized normal (N), and fullypolarized normal (NP). The FFLO phase is divided into gapped "commensurate" (C) and ungapped "incommensurate" (IC) phases. The filled circle marks the tricritical point; near it, but not visible here is a tiny region of $\mathrm{SF}_{M}$ magnetized superfluid phase, a remnant of the 3D BEC regime. The SF-NP and SF-N transitions are first-order for $\mu / \varepsilon_{B}$ above the tricritical point, along the solid heavy line. The SF-FFLO transition (solid line) is estimated from the domain wall calculation. The transition from FFLO to normal (dotted-dashed line) is assumed to be secondorder. The large circle marks the region of FFLO where $\Delta / \varepsilon_{F}$ is largest, so the phase is likely to be most robust to $T>0$ here. The dashed line near the SF-FFLO transition shows where the wave vector of the FFLO state is stationary as a function of $\mu$ : $d q / d \mu=0$ (this is calculated using the FF approximation).

of well-separated domain walls between domains where $\Delta$ is alternately positive and negative. The polarized cores of these domain walls result from occupying the spin-up Andreev bound states on each wall [21].

We calculate the energy of a single domain wall by iterating to self-consistency Eq. (5) in a finite box with periodic boundary conditions, beginning with a trial $\Delta(z)$ containing two domain walls whose separation is large compared to $\xi$. If the domain walls interact repulsively, the SF to FFLO transition is continuous and lies where this domain wall energy vanishes; otherwise this condition marks the spinodal of a first-order transition (likely to be near the true phase boundary). Within mean-field theory the transition is continuous in 1D [22], and has been argued to be so in 3D [21,23,24]: in weak-coupling the critical fields are respectively $h=(2 / \pi) \Delta_{0}\left(=0.64 \Delta_{0}\right)$, and $0.67 \Delta_{0}$, where $\Delta_{0}$ is the gap in the SF phase. We are unaware of a strong coupling $3 \mathrm{D}$ calculation of the sign of the domain wall interaction.

Figure 2 shows a representative slice of the mean-field phase diagram at fixed $t / \varepsilon_{B}=0.08$ (this slice corresponds to a fixed optical lattice intensity). Near the vacuum at small filling (low $\mu$ ) is the $3 \mathrm{D}$ BEC regime, including a very small region of the $\mathrm{SF}_{M}$ phase where the excess fermions form a Fermi liquid within the BEC. As $\mu$ and thus the filling is increased, the system crosses over towards 1D. Here, the FFLO phase appears and occupies a large portion of the phase diagram [25]. Both the SF and FFLO phases become reentrant: in the $1 \mathrm{D}$ regime the FFLO phase is at a higher $\mu$ and thus a higher density than $\mathrm{SF}$, while in the $3 \mathrm{D}$ regime this density relation is reversed. Thus, we see that the "inverted" phase separation in 1D trapped gases is connected to the standard phase separation of $3 \mathrm{D}$ via an intermediate pattern of phases where SF forms a shell surrounded by polarized phases. As $t / \varepsilon_{B}$ is further reduced, the $3 \mathrm{D}$ regime becomes smaller, with the reentrance of the SF phase moving to lower $\mu$, while the FFLO phase grows and the sliver of $\mathrm{N}$ phase between FFLO and NP is diminished. In the limit $t=0$ this phase diagram matches fairly well to that obtained from the exact solution in 1D (e.g., Fig. 1 of Ref. [11]). The main feature that the mean-field approximation misses at $t=0$ is the multicritical point where the four phases, SF, FFLO, NP and vacuum, all meet at $h=$ $-\mu=\varepsilon_{B} / 2$. In mean-field theory, the FFLO phase never extends all the way down to zero density; instead it is preempted by a first-order SF-to-NP transition.

A new $T=0$ phase transition occurs within the FFLO phase as one moves from $3 \mathrm{D}$ to $1 \mathrm{D}$ by increasing the intensity of the 2D optical lattice. In 3D the FFLO state has a Fermi surface, and is therefore gapless. In 1D the spectrum of $\mathrm{BdG}$ quasiparticles is fully gapped in the FFLO state. The gapped, commensurate FFLO state (FFLO-C) contains exactly one excess spin-up atom per 1D tube per domain wall. This commensurability means that $q=\pi b^{2}\left(n_{\uparrow}-n_{\downarrow}\right)$, while, by contrast, the number of excess up spins in the ungapped, incommensurate FFLO state (FFLO-IC) is not constrained.

The transition between FFLO-C and FFLO-IC can be understood from the band structure of the Andreev bound states on the domain walls. In FFLO-C the chemical potential lies in a gap in the quasiparticle spectrum. Thus, FFLO-C is a band insulator for the relative motion of the unpaired atoms and the condensate of pairs. As the optical lattice intensity is decreased, the 3D bands broaden and may overlap the chemical potential, opening up a Fermi surface. We approximate the IC-C transition within the FF ansatz by examining the $k_{z}>0$ half of the Fermi surface to see if it is fully gapped. In the limit $\mu / t \gg 1$, the transition occurs when $\Delta \sim 8 t h / \mu$.

We now address the question of what are the best conditions for experimentally producing, detecting and studying the FFLO phase. Ideally, one might use in situ imaging to directly observe the spatial density and magnetization modulations in this phase. In a trapped 3D gas, the modulated superfluid will occupy a hard to detect thin shell. The thinness of this shell results from the small range of $\mu$ over which the FFLO phase is stable [7]. Even approaches which produce an enlarged FFLO region in density space 
[21] share this feature. Moreover, imaging the modulations will be complicated by their 3D nature (e.g., they may form an onionlike pattern). The 1D limit also has problems. Although in 1D the FFLO phase occurs in a large region of the $T=0$ phase diagram, it has no true long-range order, only power-law correlations. Furthermore, the transition temperature $\left(T_{c}\right)$ of this $1 \mathrm{D}$ superfluid is zero. Also, for $t=0$ (strictly 1D) we have an array of independent parallel 1D clouds whose density modulations will be out of phase with one another, reducing the observed signal.

Given these concerns, we believe that the optimal conditions for observing FFLO are likely to be in the quasi-1D regime, where the $2 \mathrm{D}$ optical lattice is at an intermediate intensity that is strong enough to make the Fermi surface 1D-like (and hence enhance the instability towards FFLO), but weak enough so that atoms hopping between tubes introduce strong intertube correlations in the optical lattice. The resulting 3D long-range order can then survive to nonzero temperature [24].

Although we have only performed a $T=0$ calculation, we can crudely estimate $T_{c}$ from the size of the gap $\Delta$. For small $\Delta$, superfluid phases have $T_{c} \propto \Delta$, but when $\Delta$ approaches $\varepsilon_{F}, T_{c}$ saturates. Thus the observability of a superfluid phase such as FFLO is enhanced if the gap is increased to of order $\varepsilon_{F}$, but there is not likely to be an advantage to increasing the gap to much larger values. In 3D the maximum $T_{c} / \varepsilon_{F}$ of the SF occurs on the BEC side of the Feshbach resonance, well away from the FFLO phase [26]. However, as we move towards 1D, the FFLO phase extends more and more into the regime of strong pairing where the gap is of order $\varepsilon_{F}$, and thus we expect a large $T_{c}$. For a given $t / \varepsilon_{B}$, we find that the gap in the FFLO phase is the largest fraction of $\varepsilon_{F}$ at the SF-FFLO phase boundary near its point of reentrance, where $h$ on the SFFFLO boundary reaches its maximum (see Fig. 2). We also find that within mean-field theory this fraction $\Delta / \varepsilon_{F}$ increases as we reduce the hopping $t$. At sufficiently low $t$ the system crosses over from quasi-1D to 1D and our meanfield theory becomes unreliable. In the $1 \mathrm{D}$ limit, $T_{c}$ must vanish, so the maximum value of $T_{c} / \varepsilon_{F}$ within the FFLO phase must occur in the quasi-1D regime at some small but nonzero hopping $t$.

Another consideration that may complicate the detection of the FFLO phase within a trap is the fact that $\mu$ varies spatially both within and between tubes. This means that the local wave number $q$ of the modulation will vary through the cloud, making the modulations more difficult to detect. However, this variation can be minimized if one works near a point where $d q / d \mu=0$. We find such points do exist in the quasi-1D regime (see Fig. 2); in $3 \mathrm{D}, d q / d \mu$ is always negative so such points do not exist. Note that in the 1D limit $(t=0)$ there is even a point in the exact phase diagram near strong coupling where $d^{2} q / d \mu=d q / d \mu=$
0 that should be a real "sweet spot" for having a uniform $q$ over a fairly large fraction of a trap, and that this feature should survive to small $t$.

We thank Randy Hulet for many helpful discussions. This work was supported in part by the Army Research Office Grant No. W911NF-07-1-0464.

*Corresponding author. mparish@ @rinceton.edu

[1] G. B. Partridge et al., Science 311, 503 (2006); M.W. Zwierlein et al., Science 311, 492 (2006); M. W. Zwierlein et al., Nature (London) 442, 54 (2006); Y. Shin et al., Phys. Rev. Lett. 97, 030401 (2006); G. B. Partridge et al., Phys. Rev. Lett. 97, 190407 (2006); C. H. Schunck et al., Science 316, 867 (2007).

[2] P. Fulde and R. A. Ferrell, Phys. Rev. 135, A550 (1964).

[3] A. I. Larkin and Y. N. Ovchinnikov, Zh. Eksp. Teor. Fiz. 47, 1136 (1964) [Sov. Phys. JETP 20, 762 ( 1965)].

[4] A. Bianchi et al., Phys. Rev. Lett. 91, 187004 (2003).

[5] M. Alford, J. A. Bowers, and K. Rajagopal, Phys. Rev. D 63, 074016 (2001).

[6] T. Mizushima, K. Machida, and M. Ichioka, Phys. Rev. Lett. 94, 060404 (2005).

[7] D. E. Sheehy and L. Radzihovsky, Phys. Rev. Lett. 96, 060401 (2006); Ann. Phys. (Leipzig) 322, 1790 (2007).

[8] M. M. Parish et al., Nature Phys. 3, 124 (2007); R. Casalbuoni and G. Nardulli, Rev. Mod. Phys. 76, 263 (2004).

[9] T. Koponen et al., Phys. Rev. Lett. 99, 120403 (2007).

[10] H. Moritz et al., Phys. Rev. Lett. 94, 210401 (2005).

[11] G. Orso, Phys. Rev. Lett. 98, 070402 (2007).

[12] H. Hu, X.-J. Liu, and P. D. Drummond, Phys. Rev. Lett. 98, 070403 (2007).

[13] D. Jaksch et al., Phys. Rev. Lett. 81, 3108 (1998).

[14] M. Olshanii, Phys. Rev. Lett. 81, 938 (1998).

[15] Only 2 parameters are needed in 3D, e.g., $\mu / h, 1 / a \sqrt{2 m h}$.

[16] C. Comte and P. Nozières, J. Phys. (France) 43, 1069 (1982).

[17] P. G. de Gennes, Superconductivity of Metals and Alloys (Westview Press, Boulder, CO, 1999).

[18] J. Bardeen et al., Phys. Rev. 187, 556 (1969).

[19] We assume the normal-to-FFLO transition is still continuous for finite $t$ because the 3D limit is, at most, weakly first-order [20].

[20] R. Combescot and C. Mora, Europhys. Lett. 68, 79 (2004).

[21] N. Yoshida and S.-K. Yip, Phys. Rev. A 75, 063601 (2007).

[22] K. Machida and H. Nakanishi, Phys. Rev. B 30, 122 (1984).

[23] S. Matsuo et al., J. Phys. Soc. Jpn. 67, 280 (1998).

[24] K. Yang, Phys. Rev. B 63, 140511(R) (2001).

[25] Note that the 3D FFLO phase is absent in Fig. 2, but it is present in the 3D BCS regime when $t / \varepsilon_{B}>0.2066$.

[26] C. A. R. Sá de Melo et al., Phys. Rev. Lett. 71, 3202 (1993). 